\title{
Expression of CD44 adhesion molecules in intracranial germinomas
}

Tzuu-Yuan Huang, M.D., Jun-ichi Kuratsu, M.D., Hideo Takeshima, M.D., Toru Nishi, M.D., and Yukitaka Ushio, M.D.

Division of Neurosurgery, Kaohsiung Medical College, Kaohsiung, Taiwan; and Department of Neurosurgery, Kumamoto University Medical School, Kumamoto, Japan

Adhesion molecules play a role in tumor growth, invasiveness, and the metastatic process. The expression of CD44 adhesion molecules in 11 intracranial germinoma specimens was investigated using anti-CD44 monoclonal antibody and immunohistochemical methods. In six of 11 specimens studied, CD44 antibodies were bound to the membrane of tumor cells; in five of six specimens, CD44 antigen was also present in the cytoplasm of tumor cells. The only three patients who showed CD44-positive expression in tumor cells, lymphocytes, and extracellular matrix (ECM) exhibited either cerebrospinal fluid dissemination or multiple tumors at different locations. In all 11 specimens, no expression of CD44 in normal glial cells or capillary endothelium was detected. According to the authors' findings, the expression of CD44 in intracranial germinomas is similar to that of gonadal seminomas. Analysis of the results further suggests the possibility that the expression of CD44 in intracranial germinoma tumor cells, lymphocytes, and ECM may contribute to tumor cell migration, adhesion to cerebrospinal fluid dissemination, and/or multiple tumor locations.

Key Words * CD44 * adhesion molecule * germinoma

Adhesion molecules play important roles in cell interaction during the development and metastatic process of malignant tumors.[4,6] In the central nervous system, several adhesion molecules with specific functions involving different cells have been characterized. An adhesion molecule that belongs to the cell-surface glycoprotein family, CD44 enhances tumor growth and local invasiveness and affects the metastatic behavior of tumor cells.[4,6,17] Intracranial germinoma is characterized by its high cell migration and cerebrospinal fluid dissemination. This translocation of tumor cells may be mediated by the motility and adhesion of individual cells to extracellular matrices (ECM) and host cells.[10] The histological features of intracranial germinomas are similar to those of testicular seminoma. The expression of CD44 on seminoma biopsy samples has been reported.[7,11] We examined the expression of CD44 in intracranial germinoma specimens using immunohistochemical methods.

\section{CLINICAL MATERIAL AND METHODS}


Specimens of intracranial germinomas surgically removed from 11 patients between August 1993 and February 1997 were studied. The patients were seven males and four females who ranged in age from 10 to 39 years at the time of diagnosis (median age 19 years). Five intracranial germinomas were located in the pineal region, three in the suprasellar region, and two in the corpus callosum; in one patient, the bilateral basal ganglia were involved. Three patients had tumors at multiple sites, noted either at the time of diagnosis or developing thereafter. In two patients with relatively low Ki-67-labeling indices, marked granulomatous reaction that was positive for Masson's trichrome stain was noted. Table 1 shows the tumor locations and the clinical characteristics of our 11 patients with intracranial germinomas. All specimens were obtained at surgery, which was either stereotactic biopsy or open surgery. The 11 germinomas were diagnosed by a neuropathologist based on hematoxylin and eosin staining and immunohistochemical staining.

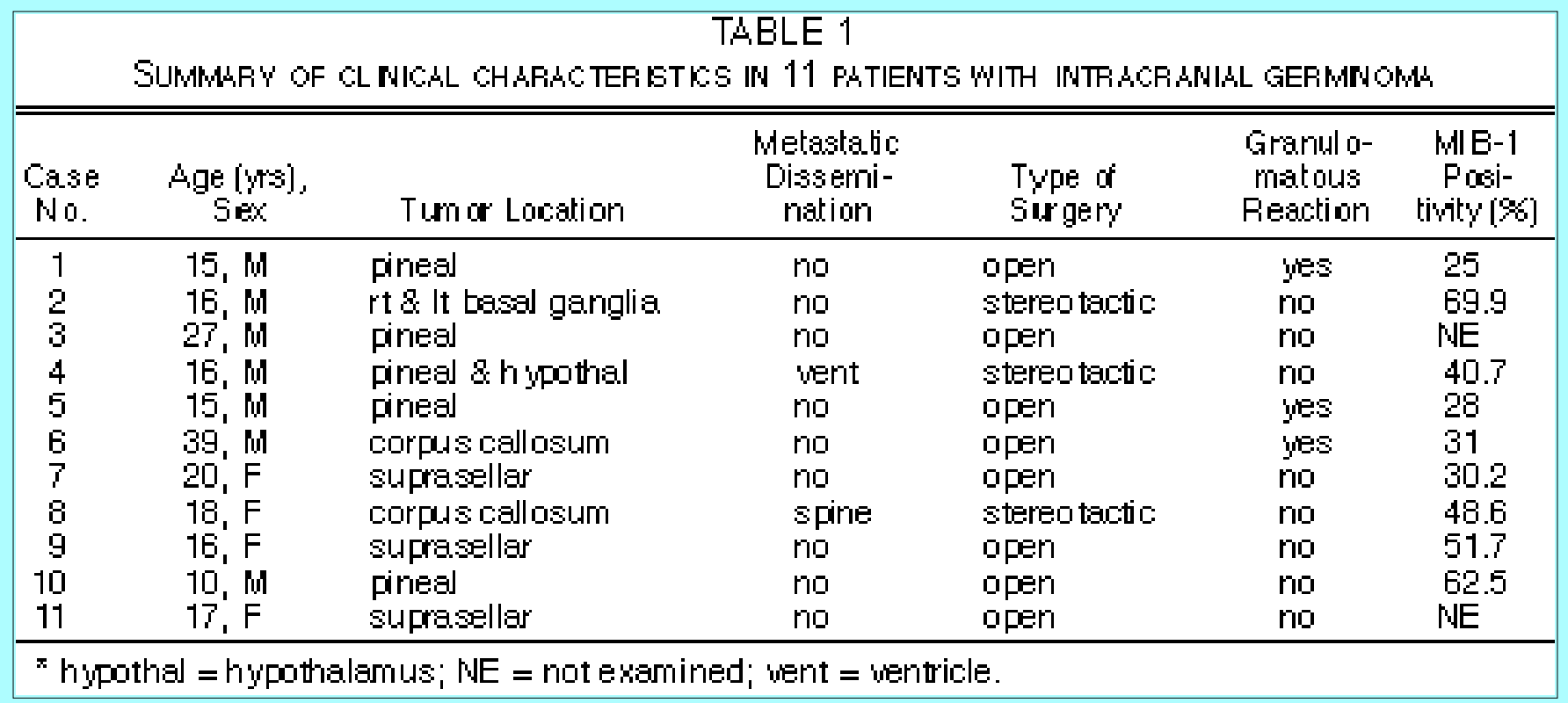

\section{Immunohistochemical Analysis}

Tumor specimens were fixed in formalin and embedded in paraffin. Thin sections $(4-5 \mu \mathrm{m})$ were prepared, deparaffinized with xylene, and rehydrated in a graded ethanol series after which $3 \%$ hydrogen peroxidase was added to eliminate endogenous peroxidase activity. The slides were covered for 1 hour at room temperature with monoclonal anti-CD44 antibodies diluted 1:50 (Novocastra Laboratories Ltd., New Castle, UK) or MIB-1 (Immunotech, Marseille, France). After three washes in phosphate-buffered saline (PBS) the tumor sections were incubated for 30 minutes at room temperature with 1:100 diluted biotinylated anti-mouse immunoglobulin G. After washing in PBS three times, the slides were reacted for 30 minutes with avidin-biotin complex and then incubated for 5 to 10 minutes in diaminobenzodine with buffer solution containing $3 \%$ hydrogen peroxidase. The slides were then counterstained with hematoxylin and observed under a microscope. The degree of CD44 immunohistochemical staining was recorded as absent (-), present $(+)$, or severe $(++)$. 


\begin{tabular}{|ccccc|}
\hline \multicolumn{5}{c|}{ TABLE 2 } \\
SummarV OF CD44 EXPRESSION & IN 11 INTRACRANIAL \\
GERMNOMA PATIENTS*
\end{tabular}

\section{RESULTS}

Table 2 summarizes the expression of CD44 in the intracranial germinoma specimens. In six specimens, CD44 antibodies were bound to the tumor cell membrane, and in five of these, CD44 antigen was found in the cytoplasm (Fig. 1 upper). In addition, we observed CD44 expression in the cell membrane of infiltrating lymphocytes in three specimens (Fig. 1 center) and the extracellular matrix (ECM) in five samples (Fig. 1 lower). Only three of 11 specimens showed CD44-positive expression in tumor cells, lymphocytes, and ECM. However, there was no expression of CD44 in adjacent normal glia cells or the capillary endothelium. A distinct granulomatous reaction with strong Masson's trichrome staining was noted in two of five specimens that showed CD44 positivity in ECM, indicating that collagen fibers were a major constituent of ECM.

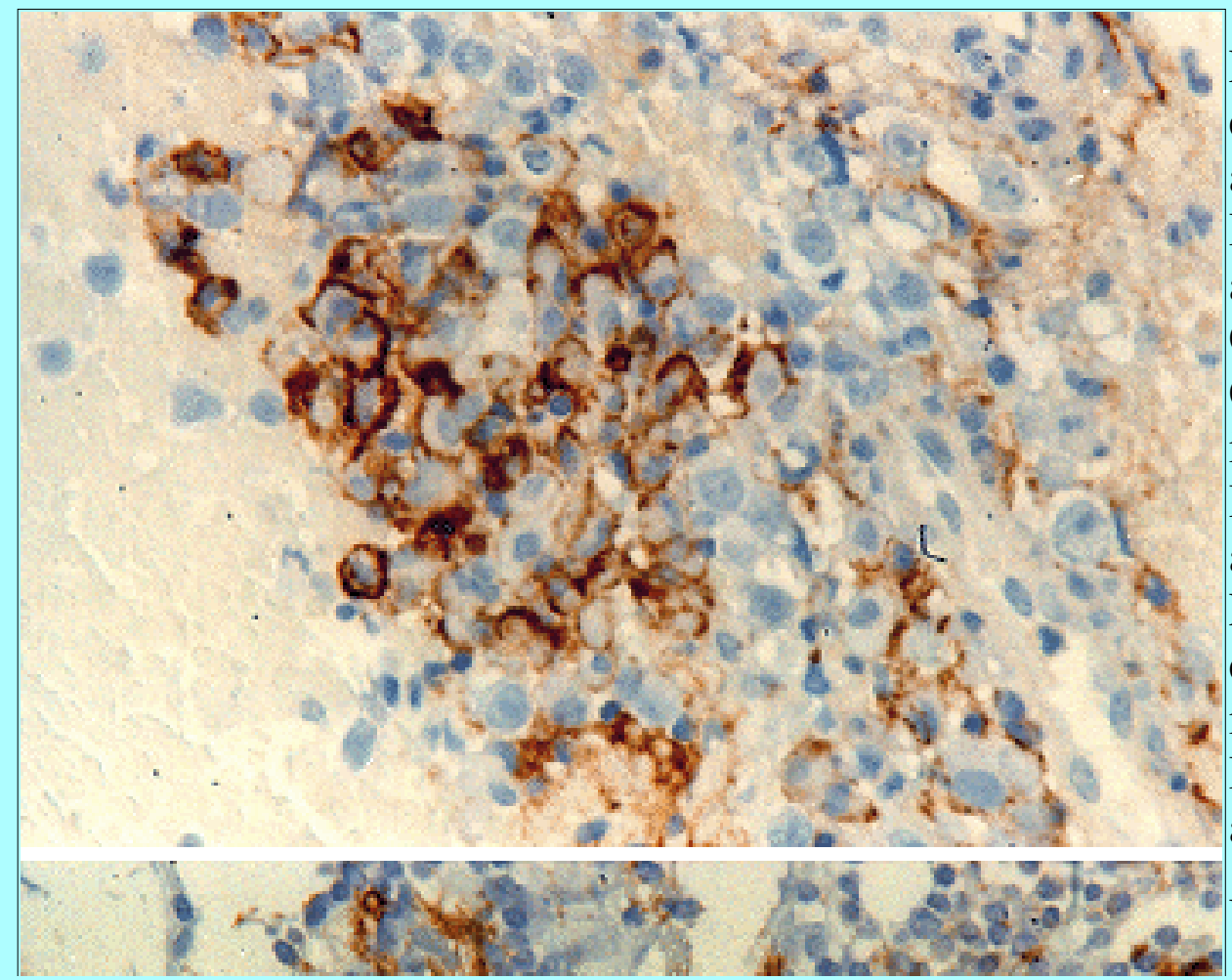

Fig. 1. Upper: Photomicrograph showing expression of CD44 in the cell membrane and cytoplasm of germinoma tumor cells. Immunohistochemical stain with CD44 antibody, original magnification X 200. Center: Photomicrograph of CD44-positive lymphocytes in an intracranial germinoma tumor specimen. Immunohistochemical stain with CD44 antibody, original magnification X 200. Lower: Photomicrograph demonstrating expression of CD44 in the ECM in an intracranial germinoma tumor specimen. Immunohistochemical stain with CD44 antibody, original magnification X 200.

Using MIB-1 monoclonal antibody, 


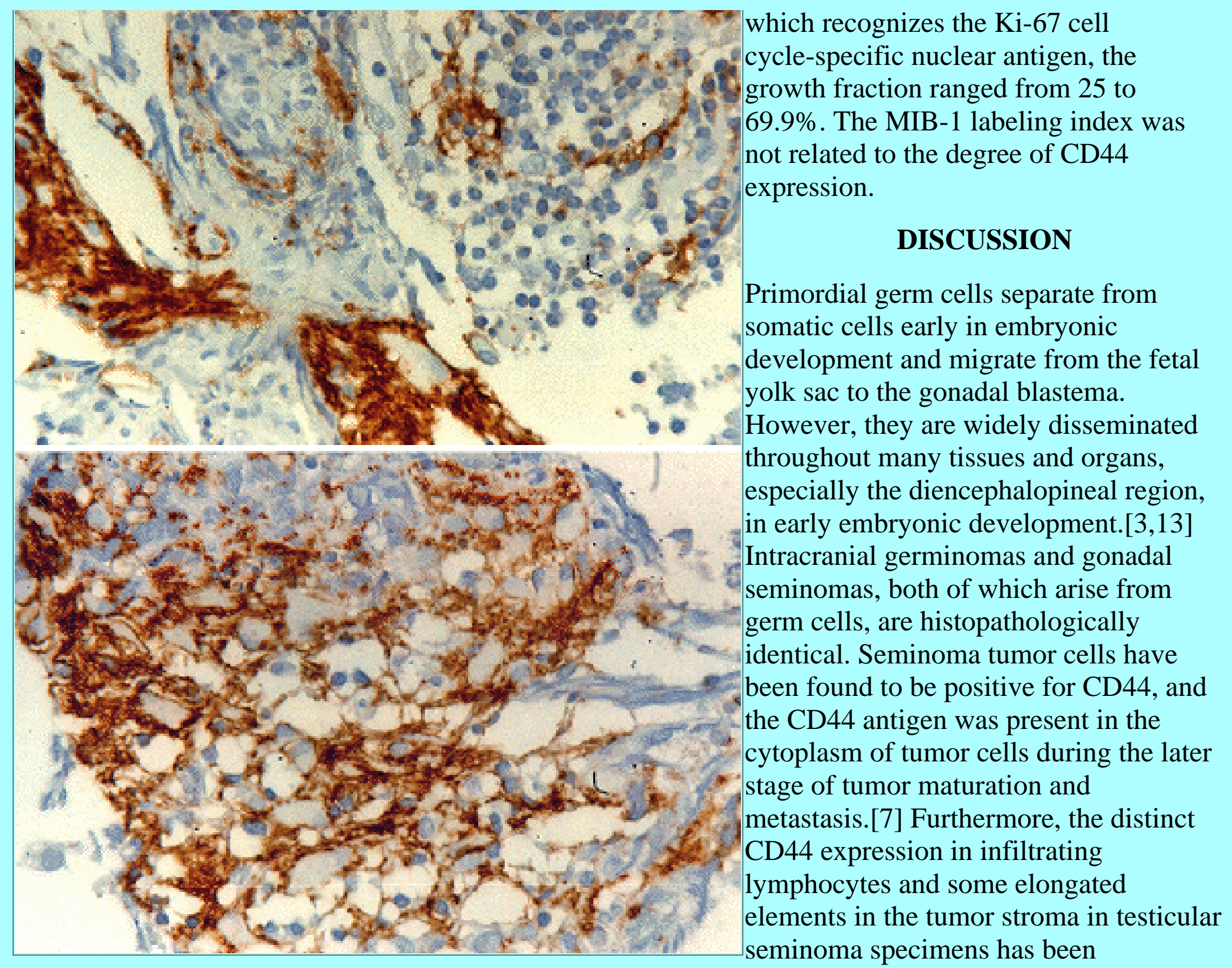

demonstrated.[11] As was the case for gonadal seminoma, in our study CD44 was expressed in the cell membrane of tumor cells, lymphocytes, and/or ECM. Therefore, intracranial germinomas and gonadal seminomas are identical with respect to their histopathological features as well as their expression of CD44.

Elevated levels of CD44 expression have been detected in carcinoma, high-grade glioma, and non-Hodgkin's lymphoma.[2,10,16] A high level of CD44 expression in non-Hodgkin's lymphoma was correlated with the clinical stage, tumor spread, and poor response to treatment.[10] Tumor growth or metastasis was suppressed by the administration of CD44 immunoglobulin fusion protein or anti-CD44 antibody,[14,18] a finding that supports the hypothesis that this molecule plays a role in tumor growth, invasiveness, and the metastatic process. Noninvasive and nonmetastatic lymphoma cells can acquire invasive as well as metastatic properties by experimentally enforced fusion with active T cells.[12] In addition to oncogenic transformation and the expression of CD44 in human cancer cells, activated lymphocytes are required for the metastatic process to occur. After antigenic activation, lymphocytes transiently express a variant of CD44. The expression and function of splice variants of CD44 appear to be essential not only for the generation of an immune response but also for the establishment of metastatic tumor colonies.[1,9] Tumor metastasis is the end result of a multistep process. In our study, 
the MIB-1 labeling index was not related to the degree of CD44 expression. Therefore, this molecule may contribute to the tumor cells' migration and adhesion rather than proliferation. Recognition of ECM components is an essential factor mediating tumor metastasis during tumor cell movement through the extracellular space. A cell-surface receptor for hyaluronate, CD44 is thought to mediate cell attachment to ECM components.[10] The expression of CD44 on the ECM may enhance tumor cell migration at several steps of the metastatic process. In our study, all three patients with tumor specimens positive for CD44 expression in tumor cells, lymphocytes, and ECM manifested either tumor dissemination or multiple tumor locations. We found that primarily the cell membrane of tumor cells or lymphocytes were stained positively. In five of our 11 cases the CD44 antigen was taken up into the cytoplasm of the tumor cells.[8] The cytoplasmic domain interacts at least indirectly with components of the cytoskeleton.[10] The ECM, filling the spaces between cells, communicates with the cell interior and modulates cell adhesion and proliferation. Positive Masson's trichrome staining of collagen fibers, a major constituent of the ECM, was noted in the intracranial germinoma specimens exhibiting a granulomatous reaction. The expression of CD44 in these specimens indicates that CD44 can interact with the ECM. Furthermore, CD44 molecules may contribute to the inflammatory process by binding T cells to endothelial cells or ECM proteins that induce or upregulate T cell activation. [5] In our study, CD44 expression in the ECM accompanied by lymphocyte infiltration may reflect the inflammatory or immune process. All of the 11 intracranial germinoma specimens showed positive expression of MT-1 in lymphocytes, suggesting that germinoma cells, both intracranial and gonadal, are endowed with an unusual capacity to contribute to the $\mathrm{T}$ cell response.

Currently available anti-human CD44 monoclonal antibody does not distinguish between the different isoforms, and immunohistochemical analyses cannot be used to predict specific tumor behavior.[15] However, the expression of CD44 in tumor cells, lymphocytes, and the ECM may point to the possibility of the tumor cell migration, adhesion in cerebrospinal fluid dissemination, or multiple tumor locations in patients with intracranial germinomas. The development of a specific anti-CD44 antibody to distinguish between different isoforms may make CD44 a good candidate to be examined for its possible contribution to metastatic dissemination.

\section{References}

1. Arch R, Wirth K, Hofmann M, et al: Participation in normal immune responses of a metastasis-inducing splice variant of CD44. Science 257:682-685, 1992

2. Aruffo A, Stamenkovic I, Melnick M, et al: CD44 is the principal cell surface receptor for hyaluronate. Cell 61:1303-1313, 1990

3. Burger PC, Scheithauer BW, Vogel FS: Surgical Pathology of the Nervous System and Its Coverings, ed 3. New York: Churchill Livingstone, 1997, pp 388-394

4. Culty M, Shizari M, Thompson EW, et al: Binding and degradation of hyaluronan by human breast cancer cell lines expressing different forms of CD44: correlation with invasive potential. J Cell Physiol 160:275-286, 1994

5. Denning SM, Le PT, Singer KH, et al: Antibodies against the CD44 p80 lymphocyte homing receptor molecule augment human peripheral blood T cell activation. J Immunol 144:7-15, 1990

6. Gunthert U, Hofmann M, Rudy W, et al: A new variant of glycoprotein CD44 confers metastatic 
potential to rat carcinoma cells. Cell 65:13-24, 1991

7. Hadziselimovic F, Herzog B, Emmons LR: The expression of CD44 adhesion molecules on seminoma cells. A new marker for early detection of the tumor. Cancer 77:429-430, 1996

8. Haynes BF, Telen MJ, Hale LP, et al: CD44--a molecule involved in lymphocyte adherence and T-cell activation. Immunol Today 10:423-428, 1989

9. Herrlich P, Zoller M, Pals ST, et al: CD44 splice variants: metastases meet lymphocytes. Immunol Today 14:395-399, 1993

10. Lesley J, Hyman R, Kincade PW: CD44 and its interaction with extracellular matrix. Adv Immunol 54:271-335, 1993

11. Rajpert-De Meyts E, Shakkebaek NE: The expression of CD44 adhesion molecules on seminoma cells. A new marker for early detection of the tumor. Cancer 78:2443-2445, 1996 (Letter)

12. Roos E, La Riviere G, Collard JG, et al: Invasiveness of T-cell hybridomas in vitro and their metastatic potential in vivo. Cancer Res 45:6238-6243, 1985

13. Russell DS, Rubinstein LJ: Pathology of Tumours of the Nervous System, ed 5. London: Edward Arnold, 1989, p 665

14. Seiter S, Arch R, Komitowski D, et al: Prevention of tumor metastasis formation by anti-variant CD44. J Exp Med 177:443-455, 1993

15. Sherman L, Sleeman J, Herrlich P, et al: Hyaluronate receptors: key players in growth, differentiation, migration and tumor progression. Cell Biol 6:726-733, 1994

16. Stamenkovic I, Amiot M, Pesando JM, et al: A lymphocyte molecule implicated in lymph node homing is a member of the cartilage link protein family. Cell 56:1057-1062, 1989

17. Sy MS, Guo YJ, Stamenkovic I: Distinct effects of two CD44 isoforms on tumor growth in vivo. J Exp Med 174:859-866, 1991

18. Sy MS, Guo YJ, Stamenkovic I: Inhibition of tumor growth in vivo with a soluble CD44-immunoglobulin fusion protein. J Exp Med 176:623-627, 1992

Manuscript received May 8, 1998.

Accepted in final form June 24, 1998.

Address reprint requests to: Tzuu-Yuan Huang, M.D., Division of Neurosurgery, Kaohsiung Medical College, Number 100, Shih-Chuan 1st Road, Kaohsiung, Taiwan, Republic of China. 